\section{Métodos alternativos en Ia construcción tradicional de los carpinteros de ribera del sur de Chile. Memoria de una práctica.}

PABLO SCHMIDT KAMP*

\section{GRADO}

GANADOR

CLASIFICACIÓN Reflexión

TEMA Construcción y materiales

DESARROLLO $\mathbf{2}^{\text {do }}$ semestre 2017

INSTITUCIÓN Universidad de Chile

PROFESOR/A Luis Goldsack

CONTACTO pabloschmidtkamp@gmail.com

\begin{abstract}
*studiante de Arquitectura de la Universidad de Chile, oriundo de la ciudad de Quillota (V región). Ha centrado su desarrollo académico en la investigación sobre la relevancia de la imprecisión en la proyectación arquitectónica. Actualmente se encuentra cursando su taller de titulación con el profesor Miguel Cassasus.
\end{abstract}

RESUMEN

La carpintería de ribera es un oficio en evidente estado de decadencia y agonía, en el que la defunción de sus antiguos artesanos ha provocado la pérdida de la cadena de enseñanza de métodos tradicionales y de memorias corporales que lo han sustentado como disciplina. El desarrollo de metodologías que asumen la ausencia y la marginalidad como dificultades inexcusables, evade la distancia que les supone su analfabetismo con estrategias que acercan a la materia la manera de operar con ella. Esta reflexión surge de la observación del trabajo artesanal dentro de los Astilleros de San Juan, Chiloé; allí se intentó comprender las lógicas constructivas que sustentan a un oficio, utilizándolo como caso de estudio. La evidente dependencia del desarrollo histórico disciplinar permite inferir que, para lograr los elementos complejos que engendra, es necesario practicar de manera reiterada para generar conocimientos materiales especialmente específicos y de carácter intangible.

palabras clave Método, Escantillón, Memoria material, Operación material, Oficio.

\section{RESUMEN}

The Seaside carpentry is a craft who passes through a visible state of agony and decadence, where the lost of its traditional craftsmen has missed the knowledge chain of its long-established methods and haptic memories which have underpinned the heritage of this profession. The develop of traditional technics that are able to assume the lackness and poverty as unavoidable realities, elude the problem that their illiteracy means, with strategies that work even closer with the material the way to operate with it. This study was completed by the observation and craftsmanship within Astilleros de San Juan, Chiloé; where the effort focused in understanding the logics that sustain the construction of a craft, using it as a case of study. The evident dependence of its own historical develop allows to conclude that to reach the complex components that generate a ship, it is necessary to be enriched by a continued trial and error process, finishing from these iterative operation specific Material memory and Haptic immaterial knowledge.

KEYwords Method, Bevel, Material memory, Material operation, Craft. 


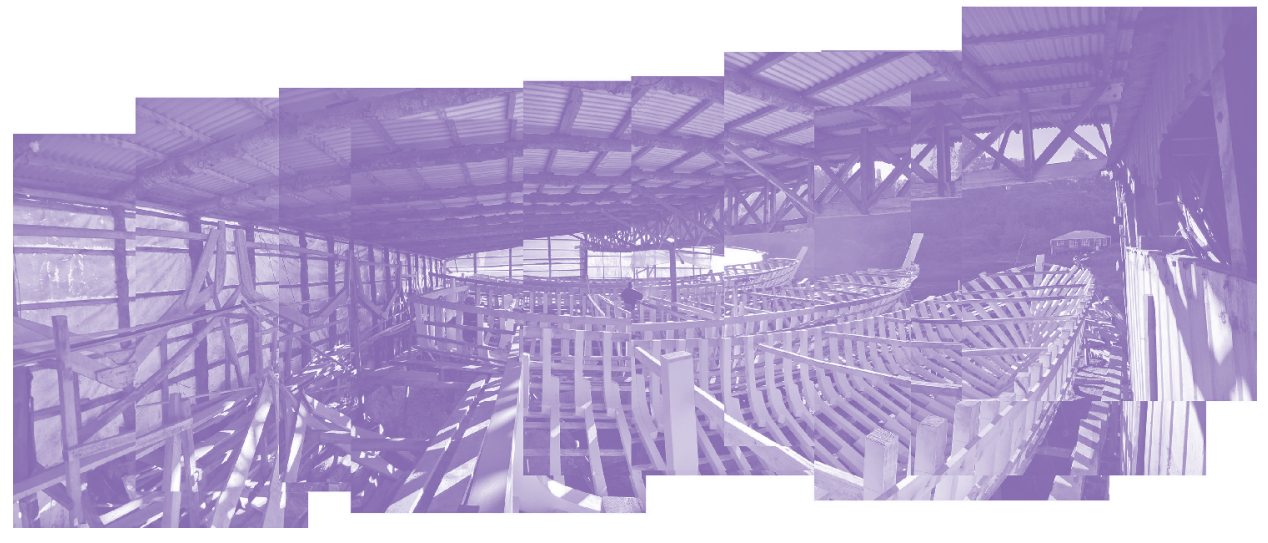

FIGURA 1.

Tres lanchas en construcción. Collage galpón exterior del astillero de Daniel Barría (San Juan, Chiloé). Elaboración propia, 2017.
Este es un oficio en agonía, porque nos referimos a una muerte anunciada, que se conoce y se comenta, y que poco se puede hacer para evitar. Sin embargo, ¿Qué es lo que desaparece como para decir que el oficio alcanzó su muerte? ¿Qué es lo que constituye esencialmente a un oficio? La carpintería de ribera en la Isla de Chiloé comenzó su proceso de decaimiento, paradójicamente, con la irrupción del desarrollo en el territorio insular [FIGURA 1]. La implementación de vías terrestres invirtió la relación de movilidad que se tenía respecto del mar y de la extracción que se tenía respecto del territorio: movilidad sobre el territorio y extracción sobre el mar. Este simple suceso, sumado a la sobreexplotación maderera en la isla, produjo un distanciamiento paulatino de la población respecto del objeto mismo de la embarcación al no ser utilizado como medio de transporte y segregarse únicamente al desarrollo extractivo y a quienes lo ejercían. Esta inversión reduce la demanda de embarcaciones y las segrega singularmente a ámbitos industriales. Para un poblado, una embarcación era un objeto directo de supervivencia y saber construirla era, para sus habitantes, una forma de sobrevivir.

La carpintería de ribera sufre una desaparición paulatina que se concreta en dos frentes: la defunción o inactividad de sus artesanos ejecutantes y el reemplazo de las técnicas tradicionales por los medios que la misma industria sostiene. Su sustento, en cambio, depende del conocimiento del artesano y el método tradicional, ya que no es posible desarrollar una embarcación si no se maneja el conocimiento.
Existen ciertas normas implícitas, reglas y variables que no se encuentran en ningún manual o libreto. Información no codificable ni traspasable sobre una hoja de papel. Esto se puede afirmar porque durante el proceso completo de la embarcación no se utilizan planos, códigos o indicaciones que definan las curvaturas, sino que esto se logra únicamente a través de la memoria del carpintero. En este caso, cualquier acción sobre la realidad involucra una operación sobre el material; si el tiempo es suficiente y dichas operaciones son sostenidas, inevitablemente se pasará a constituir un oficio, aunque no sea la intención del ejecutor. En el caso de la carpintería de ribera todo reside allí, en una prueba iterativa y extendida en el tiempo sobre la cual se han puesto a prueba las cualidades de la madera y de las operaciones que se realizaron con ella. A través de la intuición, el carpintero puede contrastar la realidad con el estándar que espera (estándar que construye iterativamente), mientras la memoria del cuerpo le permite medir (validar o rechazar) el nuevo estado de lo modificado. Es algo similar al deporte: no se aprenderá a nadar sin confrontar el agua y, para su

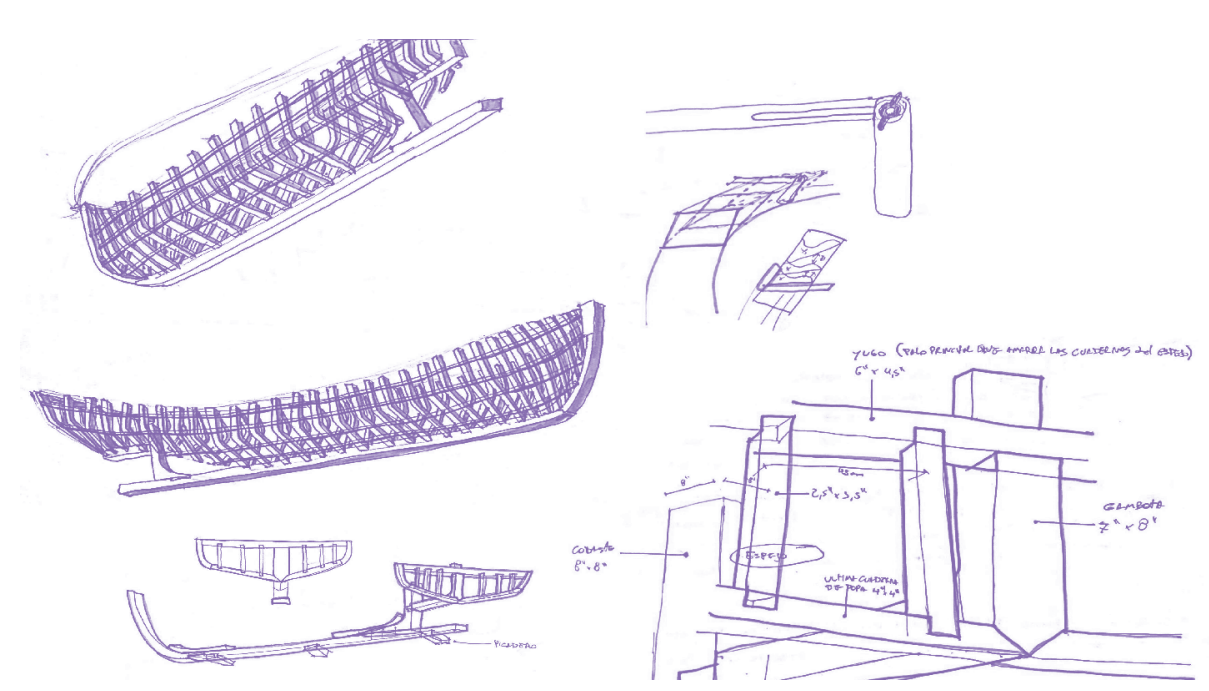

FIGURA 2.

Dibujos sobre el método y la construcción. Intento descriptivo. Elaboración propia, 2017. 

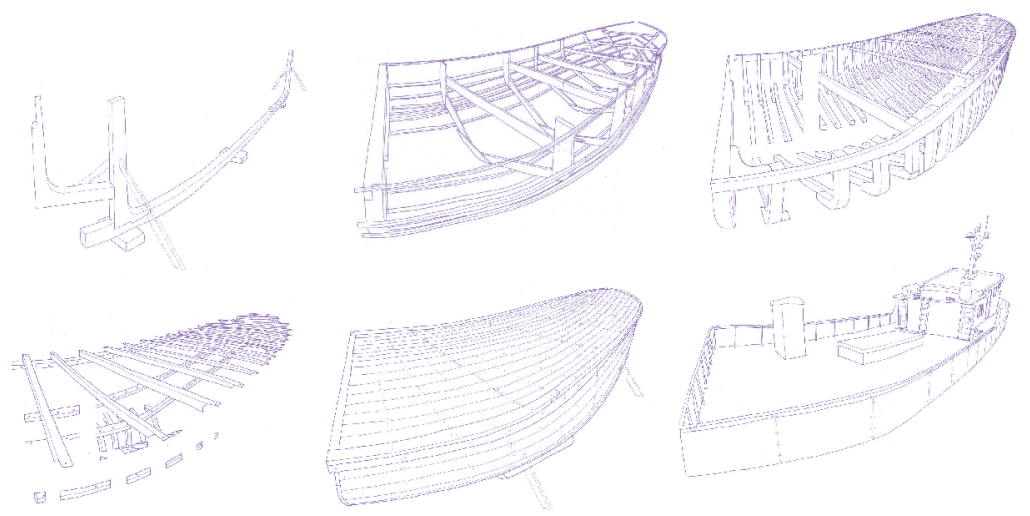

FIGURA 3.

Secuencia temporal constructiva de elementos y partes.

Método de plantillas (destacado). Elaboración propia, 2017.

perfeccionamiento, se debe exponer al cuerpo de manera reiterada a este proceso. Para aprender un oficio, además, la imitación es fundamental, ya que de allí se extrae conocimiento ya elaborado.

Es por esto que los dibujos que se realizan sobre los procesos constructivos [FIGURA 2] no son más que ilustraciones formales o indicaciones que se quedan en la especificación de elementos y partes que componen la embarcación, debido a que el conocimiento, mediante el cual se puede concebir una embarcación, reside en la calibración ocular para medir visualmente una curvatura, la noción tangible de resistencia del material respecto al esfuerzo al que será sometido y la curvatura posible por un listón de madera medida a través del tacto. De ahí que Daniel Barría, carpintero de ribera de San Juan, Chiloé, señale como fundamental el haberse sometido a la experiencia corporal del navegante. Todas las memorias corporales interiorizadas se hacen evidentes a través de la interacción material. Sin embargo, ilustrar o dibujar sobre una hoja permite describir, o al menos acercarse, al segundo componente relevante en la constitución del oficio: el método.

Si bien un oficio se conforma por la reiteración operativa sobre un material, la manera en que se ordena dicha operación, es decir, las reglas procedimentales que definen el método, sí constituyen un factor que determina un oficio y lo ubica en un campo determinado, ya que la composición material y las reglas que apresan su propia lógica constructiva son factores relevantes tanto para el proceso como para el objeto final elaborado. Sobre todo, el método es relevante cuando se contextualiza la realidad educacional marginal que poseen quienes ejecutan y dirigen la obra, encontrándose entre ellos un estado de total analfabetismo. ¿Cómo es posible conseguir una geometría excesivamente compleja sin siquiera leer los números de una huincha?

El método de plantillas [FIGURA 3] se fundamenta en la elaboración del volumen total del casco de la embarcación mediante una estructura temporal conformada por planti-

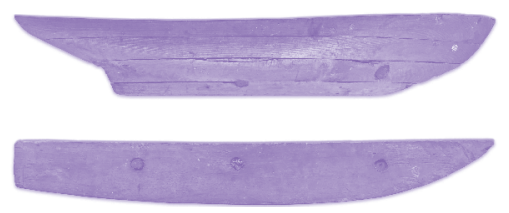

FIGURA 4.

Maqueta elaborada por Alfonso Bahamonde en el año 1957 (Carpintero de ribera retirado). Imagen elaboración propia, 2017. llas (guías de curvatura ubicadas en sentido transversal) y cintas (listones de alta esbeltez ubicados en sentido longitudinal). Estos elementos son los más importantes a lo largo del proceso constructivo, ya que permiten medir la curvatura, establecer puntos de apoyo para desplazarse sobre la obra, constituir la figura del casco para ser corregida y elaborar la totalidad de las piezas que lo componen.

Este método de plantillas se sustenta en una herramienta particular que permite medir las múltiples curvaturas o dobles curvaturas, divergentes entre sí, que posee cada pieza de la embarcación. El escantillón [FIGURA 2] es un medidor de magnitudes angulares con forma de compás que permite establecer su inclinación entre la hoja metálica y el cuerpo de madera para corresponder materialmente (no numéricamente) la magnitud angular exacta para cada porción de pieza, midiendo directamente desde el volumen temporal previamente construido. Dimensionar una porción específica de manera material permite operar con ella: traspasarla, moverla y replicarla en otra pieza de madera para producir los elementos que finalmente componen una lancha. Sería totalmente imposible, o de una complejidad indescriptiblemente engorrosa, intentar desarrollar cada una de las piezas y sus correspondientes curvaturas mediante indicaciones numéricas. Su cantidad es indeterminable y su posición difícil de corresponder únicamente con medidas. Este simple paso del número al material basado en una pobreza metodológica extrema se hace fundamental para lograr concebir que un analfabeto alcance tal resolución constructiva de la manera más material posible.

Otro método, menos popular debido a su irrupción dentro de la complejidad numérica, es el de maqueta y tarima [FIgURA 4] que, como su nombre lo dice, comienza con la elaboración de un modelo a escala conformado por tablas calibradas superpuestas en sentido vertical que se une como conjunto a través de tarugos. Este método permite obtener modelos distintos o diseños específicos de embarcaciones, ya que mediante la modificación en el modelo es 

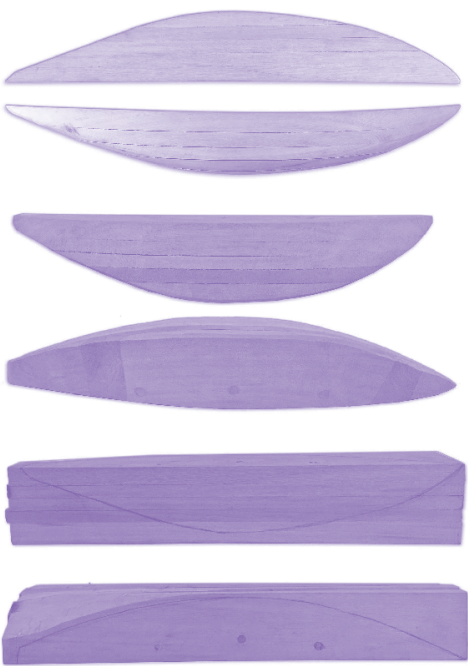

FIGURA 5.

Proceso de factura de modelo para un velero de $8 \mathrm{~m}$. Elaboración del autor. Imagen elaboración propia, 2017.

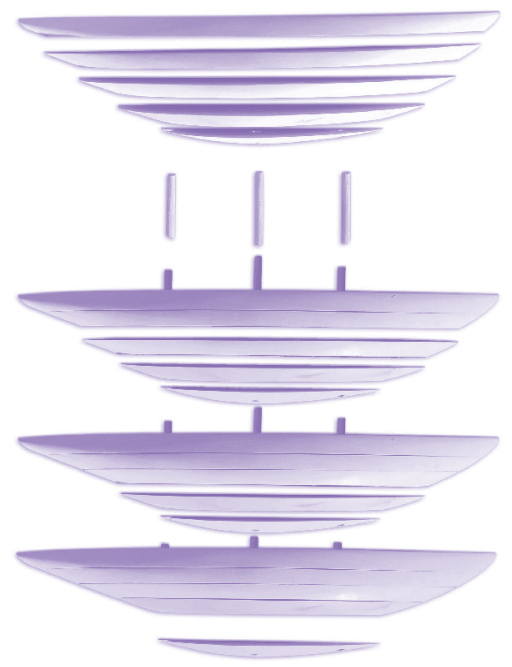

FIGURA 6.

Proceso de desarme de modelo para un velero de $8 \mathrm{~m}$. Elaboración del autor. Imagen elaboración propia, 2017. rederos directos de una larga cadena, seguramente común, que se encargó de testear una selección natural intervenida artificialmente. Esta subordinación reiterada del objeto somete cualquier avance en su elaboración a una prueba de resistencia que, al sostenerse en el tiempo, complejiza paulatina y discretamente el resultado hacia un objeto cada vez más elaborado.

En las orillas de la playa de San Juan se olvidan restos oxidados de piezas metálicas que han dejado los procesos de reparación. Elementos a los que no se les adjudica una procedencia conocida y que las aguas salinas han modificado. Como búsqueda paralela y aplicada, se operó materialmente de manera reiterada sin someter estas estructuras a un esfuerzo más que a la posible variar el resultado del objeto final. Esta maqueta se elabora [FIGURA 5] a partir de un conjunto compuesto de las tablas ya entarugadas y calibradas a una escala, permitiendo el desarme [FIGURA 6] y traspaso de las curvaturas pertenecientes a cada porción de madera a un plano [FIGURA 7]. Estos modelos evitan un problema fundamental para las embarcaciones de manera extremadamente simple.

La simetría de la embarcación es asegurada mediante la elaboración de la mitad del modelo, lo que obliga a replicar de manera espejada cada una de las piezas que se elaboran y da, a quien confecciona la maqueta, una única y vital superficie lisa para apoyar y trabajar con el modelo. Uno de los puntos débiles de este método es que, al definir la figura de la embarcación definitiva con un modelo a escala, cualquier imprecisión en el modelo se ve exacerbada al momento de construir la embarcación definitiva, por lo que la memoria corporal, que se expresa en la minuciosidad y claridad de quien la ejecuta, se torna fundamental a la hora de concebir un resultado de acuerdo al estándar esperado.

La claridad y excesiva complejidad que es posible apreciar en los métodos utilizados difiere de la posibilidad de que hayan sido alcanzados por medio de inspiraciones o chispazos del ingenio. Se torna imposible asimilar, dentro de un solo objeto, la inconmensurable cantidad de decisiones concordantes; ellas no pueden depender de una eficiencia regida por estándares ingenieriles. La historia de construcciones náuticas se remonta a tiempos inmemorables y los modelos que se construyen en San Juan (Chiloé) son he-

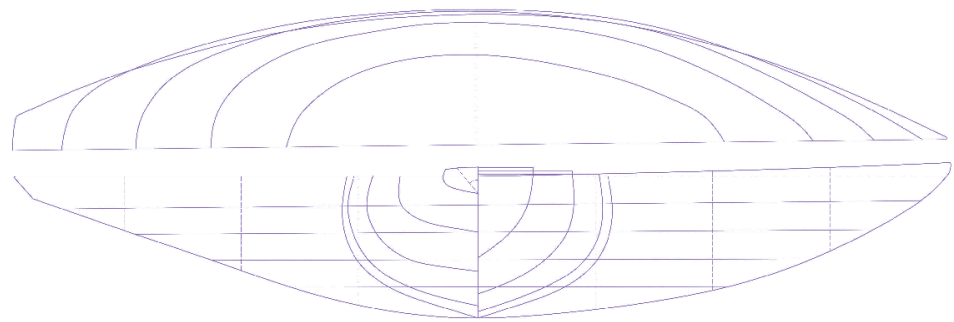

FIGURA 7.

Planimetría sin escala del modelo para un velero de $8 \mathrm{~m}$. Elaboración del autor. Imagen elaboración propia, 2017. 

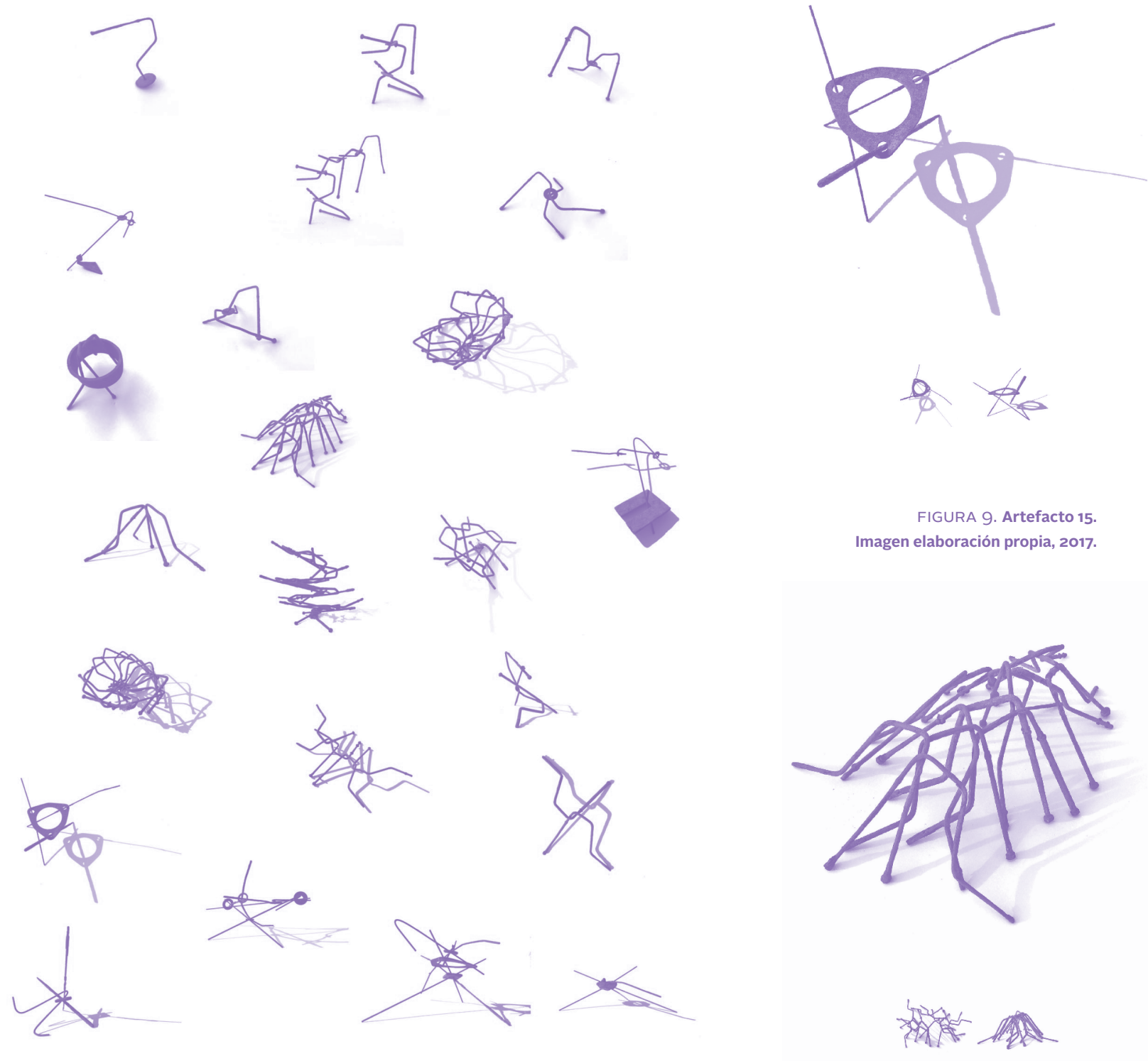

FIGURA 8. Catálogo de artefactos. Elaboración del autor. Imagen elaboración propia, 2017.

FIGURA 10. Artefacto 23 Imagen elaboración propia, 2017.

NOTA: El objetivo real de este escrito nunca estuvo completamente claro, quizás el devenir de las circunstancias vividas moldean de manera exagerada lo que escribo. Nunca tuve un asiento, un escritorio o algún acompañante continuado durante este tiempo, sólo el ejercicio didáctico de una conversación crítica con algún sensato interpelador esporádico o la simple compañía de mis pensamientos. No pretendo encontrar verdades inmutables ni seguridad en mis planteamientos, simplemente intento conducir mis ánimos hacia la comprensión de la asombrosa complejidad de lo existente e imprecisión en lo real con la insistente porfía de hundir los dedos en la llagas del material. 